\title{
Factors affecting cognitive function according to gender in community-dwelling elderly individuals
}

\author{
Miwon Kim', Jeong-Mo Park² \\ 'Department of Nursing Science, Sangmyung University, Cheonan, Korea; '2Department of Nursing, Kyungin Women's University, Incheon, Korea
}

\begin{abstract}
OBJECTIVES: This study aimed to identify the factors affecting the cognitive function of elderly people in a community by gender.

METHODS: We obtained 4,878 secondary data of people aged $\geq 65$ years in 2016 at a dementia prevention center in Gyeyang-gu, Incheon. Data were obtained through Mini-Mental Status Examination optimized for screening dementia and a questionnaire. The data were statistically analyzed using analysis of variance, analysis of covariance, and hierarchical regression.
\end{abstract}

RESULTS: There were significant differences in cognitive function according to gender, and the differences were significant even when age was controlled, but gender differences disappeared when education was controlled. Age, education, social activities, number of comorbid diseases, and alcohol drinking affected cognitive function through interaction with gender, but interaction with gender disappeared when education was controlled. Regression analysis showed that depression, cohabitant, social activities etc., had a significant impact on both men and women under controlled education and age. In men, the effect of social activities was greater than that of women, and hyperlipidemia had the effect only in women.

CONCLUSIONS: The differences in gender-related cognitive functions were due to differences in gender education period. The period of education is considered to have a great influence on cognitive function in relation to the economic level, occupation, and social activity.

KEY WORDS: Cognitive function, Elderly, Gender, Korea

\section{INTRODUCTION}

In 2000, the population aged 65 years and older in South Korea (hereafter Korea) exceeded $7 \%$ of the total population and entered the aging society. As of 2017 , the elderly population already exceeded $13.8 \%$ [1] and the elderly population is still rapidly growing. The rapid aging of the population has social and economic

\section{Correspondence: Miwon Kim}

Department of Nursing Science, Sangmyung University,

31 Sangmyeongdae-gil, Dongnam-gu, Cheonan 31066, Korea

E-mail:kmw@smu.ac.kr

Received: Oct 10, 2017 / Accepted: Nov 5, 2017 / Published: Nov 15, 2017

This article is available from: http://e-epih.org/

(C) This is an open-access article distributed under the terms of the Creative Commons Attribution License (http://creativecommons.org/licenses/by/4.0/), which permits unrestricted use, distribution, and reproduction in any medium, provided the original work is properly cited.

(C) 2017, Korean Society of Epidemiology cost implications, resulting in personal and social burdens. Aging is also a direct cause of many degenerative diseases, including dementia, the most serious neurodegenerative disease, which our society has to face due to aging [2]. According to the 3rd Dementia management integration plan, the number of patients with dementia is expected to exceed 1 million in 2024 and 2 million in 2014 [3]. Also, the annual rate of progression to mild cognitive impairment (MCI) in normal subjects has been estimated at between $1-4 \%$ annually, and subjects with MCI have an annual risk of $12 \%$ of developing dementia [4]. The decline in cognitive function due to aging progresses slowly, making it difficult to pathologically determine the exact timing of onset of dementia. However, once it has progressed to dementia, it causes deterioration in the quality of life of patients and their families and huge medical expenses, leading to high economic burden at the individual and national levels. Therefore, countermeasures against this are urgently needed [2]. Since no effective drug and treatment for cognitive impairment and dementia is currently available, early detection of 
modifiable risk factors for cognitive impairment and prevention of cognitive impairment and delaying the onset of dementia through related early intervention is an important task of the national dementia management project [2]. In line with this, the Dementia screening project for the early detection of dementia has intensively been implemented at public health centers nationwide since 2010. As a result, the number of patients with mild cognitive impairment undergoing treatment increased from 24,602 in 2010 to 105,598 in 2014 with an annual increase rate of $43.9 \%$ [5].

Factors affecting cognitive impairment that have been identified so far include age, educational period, gender [6-10], health life factors such as drinking and smoking [7], depression [11], social factors such as social activity and occupation, history of disease, and body mass index (BMI) [12]. However, among these factors, age, education, and depression are consistently reported as risk factors for cognitive impairment, but these factors are not all consistent among studies. When cognitive decline is regarded as a continuous process from normal cognitive function to mild cognitive impairment and dementia, the identification and management of influential factors such as cognitive decline-related demographic characteristics, comorbid diseases and health habits may contribute to the delay or prevention of dementia [6]. However, previous studies on prediction models for cognitive function have been conducted in patients with cognitive impairment or dementia; thus, there is a limitation to generalizing the results of those studies [6]. In order to determine factors for preventing cognitive decline, it is also necessary to conduct studies involving the entire elderly population living in the community, including the elderly with normal cognitive function and cognitive decline.

Cognitive decline is expected in all elderly people rather than a specific group, and those with cognitive decline have a wide range of characteristics in addition to demographic characteristics including gender. Therefore, it is difficult to see that cognitive decline factors identified in the whole elderly population may have the same effects in the sub-elderly group. In order to prevent cognitive decline more effectively, it is necessary to manage factors which were found to be significantly associated with cognitive decline depending on the target population. The basic distinction in establishing intervention plans suited for target participants is gender, and it is thus necessary to identify factors affecting cognitive decline according to gender and to establish intervention plans based on such a gender difference. Many previous studies investigating factors for the cognitive decline have reported that a difference prevalence of cognitive decline in men and women was [69,10,13]. Kim et al. [14] compared cognitive function using the Korean version of Mini-Mental Status Examination optimized for screening dementia (MMSE-DS), the Korean version of the Consortium to Establish a Registry for Alzheimer's Disease Assessment Packet(CERAD-K) and Korean-MMSE (K= MMSE) and reported that gender among education period, age and gender was found to have the greatest effect on cognitive function as measured by all the tools. However, studies identifying differences in influential factors according to gender are scarce and the results are inconsist- ent. Women are reported to have more cognitive decline than men $[6,7]$.

Therefore, the present study aimed to investigate factors affecting cognitive function in the community-dwelling elderly, to identify factors affecting cognitive decline according to gender.

\section{MATERIALS AND METHODS}

\section{Study design and participants}

This is a cross-sectional study designed to investigate factors affecting cognitive function in the elderly using data obtained from elderly individuals aged 65 years or older who underwent early dementia screening at a dementia prevention center located in Gyeyang-gu, Incheon, Korea in 2016. The participants underwent dementia screening by examiners who visited homes, community centers and senior citizen centers.

\section{Instruments \\ Cognitive function-related variables}

Age, gender, educational period, economic status, social life (past occupation, number of social activities, religion, cohabitant), history of disease (diabetes, hypertension, stroke, hyperlipidemia, number of comorbid diseases), and health habits (drinking, smoking, exercise) were examined.

\section{Cognitive screening tool}

Cognitive function state was examined using the MMSE-DS [14]. The reliability of this tool, as reported by Kim et al. [14] was Cronbach's $\alpha=0.826$, and its reliability in the present study was Cronbach's $\alpha=0.839$.

\section{Depression scale}

The degree of depression was assessed using the Short Form Geriatric Depression Scale (S-GDS)-Korean version of the 15item S-GDS originally developed by Sheikh \& Yesavage [15]. The Korean version was translated and standardized by Ki [16]. The scores range from 0 to 15 points on a 2-point scale ( 1 point for 'yes' and 0 point for 'no'), and a higher score indicates a higher degree of depression. The degree of depression is classified as normal state for a score of less than 4 points, mild depression for a score of 5-8 points and severe depression for a score of more than 9 points. Its reliability in a study by Ki [16] was Cronbach's $\alpha=0.884$.

\section{Data collection}

For secondary data analysis, the present study obtained the consent from the head of the institution for the scope and contents of the data and was approved by the official institutional review board (IRB) designated by the Ministry of Health and Welfare Affairs (IRB no. PO1-201703-21-019). Data were collected through dementia screening as part of an early dementia screening, publicity and education project at a dementia prevention center from December 2015 to the end of December 2016. Dementia screening was performed by 4 nurses who completed a specialized dementia 
education and were systemically educated about the screening method. After all the participants were briefed about the purpose and contents of the screening and the present study and provided their consent for the use of the screening results, the screening and data collection were performed in a quiet place on a 1:1 basis.

\section{Data analysis}

The data were obtained from 4,878 participants with complete entries among those who received dementia screening, and were analyzed using SPSS version 18.0 (SPSS Inc., Chicago, IL, USA). Differences in gender distribution of all the variables were ana-

Table 1. Distribution of cognitive-related variables by gender

\begin{tabular}{|c|c|c|c|c|c|c|}
\hline Variables & Categories & Total & Men & Women & $\chi^{2}$ & $p$-value \\
\hline Gender & & $4,878(100.0)$ & $1,110(22.8)$ & $3,768(77.2)$ & & \\
\hline \multirow[t]{3}{*}{ Age } & $<74$ & $1,868(38.3)$ & 475 (42.8) & $1,393(37.0)$ & 723.30 & $<0.001$ \\
\hline & $75-84$ & $2,351(48.2)$ & $519(46.8)$ & 1,832 (48.6) & & \\
\hline & $\geq 85$ & $659(13.5)$ & $116(10.5)$ & $543(14.4)$ & & \\
\hline \multirow[t]{4}{*}{ Education period (yr) } & $0-3$ & $1,803(37.0)$ & $113(10.2)$ & $1,690(44.9)$ & 13.58 & $<0.001$ \\
\hline & $4-6$ & $1,519(31.1)$ & $321(28.9)$ & $1,198(31.8)$ & & \\
\hline & $7-12$ & $1,365(28.0)$ & $552(49.7)$ & $813(21.6)$ & & \\
\hline & $\geq 13$ & $188(3.9)$ & $124(11.2)$ & $64(1.7)$ & & \\
\hline \multirow[t]{3}{*}{ Depression } & Normal & $3,664(75.1)$ & $830(74.8)$ & $2,834(75.2)$ & 1.66 & 0.44 \\
\hline & Mild depression & $638(13.1)$ & $138(12.4)$ & $500(13.3)$ & & \\
\hline & Severe depression & $576(11.8)$ & $142(12.8)$ & $434(11.5)$ & & \\
\hline \multirow[t]{2}{*}{ Past occupation } & Never & $2,819(57.8)$ & $179(16.1)$ & $2,640(70.1)$ & $1,022.60$ & $<0.001$ \\
\hline & Yes & 2059 (42.2) & 931 (83.9) & $1,128(29.9)$ & & \\
\hline \multirow[t]{3}{*}{ No. of social activities } & 0 & $1,026(21.0)$ & $335(30.2)$ & $691(18.3)$ & 92.69 & $<0.001$ \\
\hline & 1 & $2,972(60.9)$ & $640(57.7)$ & $2,332(61.9)$ & & \\
\hline & $\geq 2$ & $880(18.0)$ & $135(12.2)$ & $745(19.8)$ & & \\
\hline \multirow[t]{2}{*}{ Religion } & No & $1,665(33.0)$ & $533(46.2)$ & $2,636(30.0)$ & 127.98 & $<0.001$ \\
\hline & Yes & $3,213(67.0)$ & $577(53.8)$ & $574(70.0)$ & & \\
\hline \multirow[t]{3}{*}{ Cohabitant } & Alone & $1,514(31.0)$ & 209 (18.8) & 1,305 (34.6) & 593.98 & $<0.001$ \\
\hline & Spouse & $1,917(39.3)$ & $781(70.4)$ & $1,136(30.1)$ & & \\
\hline & Non-spouse & $1,447(29.7)$ & $120(10.8)$ & $1,327(35.2)$ & & \\
\hline \multirow[t]{2}{*}{ ES } & National Health Insurance & $4,604(94.4)$ & $1,031(92.9)$ & $3,573(94.8)$ & 6.28 & 0.01 \\
\hline & Medical Aid & $273(5.6)$ & $79(7.1)$ & $194(5.1)$ & & \\
\hline \multirow[t]{2}{*}{ Hypertension } & No & $2,030(41.6)$ & $575(51.8)$ & $1,455(38.6)$ & 61.06 & $<0.001$ \\
\hline & Yes & $2,845(58.4)$ & $535(48.2)$ & $2,310(61.3)$ & & \\
\hline \multirow[t]{2}{*}{ DM } & No & $3,664(75.1)$ & $839(75.6)$ & $2,825(75.0)$ & 0.17 & 1.00 \\
\hline & Yes & $1,214(24.9)$ & $271(24.4)$ & $943(25.0)$ & & \\
\hline \multirow[t]{2}{*}{ Hyperlipidemia } & No & $4,084(83.7)$ & $1,008(90.8)$ & $3,076(81.6)$ & 52.98 & $<0.001$ \\
\hline & Yes & $794(16.3)$ & $102(9.2)$ & $692(18.4)$ & & \\
\hline \multirow[t]{2}{*}{ Stroke } & No & $4,641(95.1)$ & $1,052(94.8)$ & $3,589(95.2)$ & 0.42 & 0.52 \\
\hline & Yes & $237(4.9)$ & $58(5.2)$ & $179(4.7)$ & & \\
\hline \multirow[t]{3}{*}{ No. of comorbid diseases } & 0 & $833(17.1)$ & $255(23.0)$ & $578(15.3)$ & 51.78 & $<0.001$ \\
\hline & $1-2$ & $3,209(65.8)$ & $716(64.5)$ & $2,493(66.2)$ & & \\
\hline & $\geq 3$ & $836(17.1)$ & 139 (12.5) & $697(18.5)$ & & \\
\hline \multirow[t]{3}{*}{ Alcohol drinking } & Never & $3,618(74.2)$ & $239(21.5)$ & $3,379(89.7)$ & $2,105.34$ & $<0.001$ \\
\hline & Ceased drinking & $552(11.3)$ & $420(37.8)$ & $132(3.5)$ & & \\
\hline & Currently drinking & 708 (14.5) & $451(40.6)$ & $257(6.8)$ & & \\
\hline \multirow[t]{3}{*}{ Smoking } & Never & 3,995 (81.9) & $329(29.6)$ & $3,666(97.3)$ & $2,659.31$ & $<0.001$ \\
\hline & Ceased smoking & 705 (14.4) & $641(57.7)$ & $64(1.7)$ & & \\
\hline & currently smoking & $178(3.6)$ & 140 (12.6) & $38(1.0)$ & & \\
\hline \multirow[t]{2}{*}{ Exercise } & No & $2,264(46.4)$ & $418(37.7)$ & $1,846(49.0)$ & 44.28 & $<0.001$ \\
\hline & Yes & $2,614(53.6)$ & 692 (62.3) & $1,922(51.0)$ & & \\
\hline
\end{tabular}

Values are presented as number (\%).

$\mathrm{ES}$, economic status by health insurance type; DM, diabetes mellitus. 
lyzed using real numbers, percentages and chi-square test. Cognitive function by gender for each variable was analyzed using $t$-test and analysis of variance (ANOVA), the interaction of each varia- ble with gender was analyzed using two way ANOVA, and the interactions of each variable with gender when age and education was controlled were analyzed using analysis of covariance. The

Table 2. Gender comparison of cognitive function score differences by variables

\begin{tabular}{|c|c|c|c|c|c|c|c|}
\hline \multirow{2}{*}{ Variables } & \multirow{2}{*}{ Categories } & \multicolumn{2}{|c|}{ Total } & \multicolumn{2}{|c|}{ Men } & \multicolumn{2}{|c|}{ Women } \\
\hline & & Mean $\pm S D$ & tor $F$ & Mean \pm SD & tor $F$ & Mean \pm SD & tor $F$ \\
\hline \multirow[t]{2}{*}{ Gender } & Men & $25.53 \pm 3.72$ & $54.31^{* * *}$ & & & & \\
\hline & Women & $23.76 \pm 4.47$ & & & & & \\
\hline \multirow[t]{3}{*}{ Age } & $<74$ & $25.17 \pm 3.41$ & $303.29 * * *$ & $26.32 \pm 3.20$ & $25.161^{* * *}$ & $25.56 \pm 2.46$ & $202.99 * * *$ \\
\hline & $75-84$ & $26.64 \pm 4.32$ & & $25.04 \pm 3.84$ & & $23.25 \pm 4.37$ & \\
\hline & $\geq 85$ & $23.16 \pm 4.60$ & & $24.25 \pm 4.34$ & & $20.86 \pm 5.06$ & \\
\hline \multirow[t]{4}{*}{ Education period (yr) } & $0-3$ & $21.66 \pm 4.49$ & $458.35^{* * *}$ & $22.73 \pm 4.15$ & $49.99 * * *$ & $21.58 \pm 4.50$ & $342.63^{* * *}$ \\
\hline & $4-6$ & $24.71 \pm 3.94$ & & $24.53 \pm 4.28$ & & $24.76 \pm 3.84$ & \\
\hline & $7-12$ & $26.47 \pm 2.89$ & & $26.40 \pm 2.97$ & & $26.52 \pm 2.84$ & \\
\hline & $\geq 13$ & $26.92 \pm 2.66$ & & $25.53 \pm 3.72$ & & $27.13 \pm 2.82$ & \\
\hline \multirow[t]{3}{*}{ Depression } & Normal & $24.62 \pm 4.00$ & $95.83^{* * *}$ & $26.00 \pm 3.18$ & $29.00 * * *$ & $24.21 \pm 4.12$ & $73.89^{* * *}$ \\
\hline & Mild & $23.23 \pm 4.68$ & & $24.43 \pm 4.43$ & & $22.89 \pm 4.69$ & \\
\hline & Severe & $22.00 \pm 5.67$ & & $23.69 \pm 5.16$ & & $21.45 \pm 5.72$ & \\
\hline \multirow[t]{2}{*}{ Past occupation } & Never & $23.40 \pm 4.64$ & $208.76^{* * *}$ & $24.53 \pm 4.69$ & $15.71^{* * *}$ & $23.33 \pm 4.63$ & $83.18^{* * *}$ \\
\hline & Yes & $25.20 \pm 3.73$ & & $25.73 \pm 3.47$ & & $24.76 \pm 3.88$ & \\
\hline \multirow[t]{3}{*}{ No. of social activities } & 0 & $23.90 \pm 4.95$ & $2.70^{+}$ & $24.84 \pm 4.23$ & $5.78^{* * *}$ & $23.44 \pm 5.20$ & 2.85 \\
\hline & 1 & $24.26 \pm 4.24$ & & $25.86 \pm 3.33$ & & $23.82 \pm 4.36$ & \\
\hline & $\geq 2$ & $24.13 \pm 4.07$ & & $25.71 \pm 3.92$ & & $23.84 \pm 4.04$ & \\
\hline \multirow[t]{2}{*}{ Religion } & No & $24.29 \pm 4.43$ & $10.58^{* * *}$ & $25.53 \pm 4.09$ & 0.03 & $24.02 \pm 4.13$ & $8.56^{* *}$ \\
\hline & Yes & $23.61 \pm 4.44$ & & $25.54 \pm 3.68$ & & $23.13 \pm 4.69$ & \\
\hline \multirow[t]{3}{*}{ Cohabitant } & Alone & $23.99 \pm 4.14$ & $206.27^{* * *}$ & $25.35 \pm 3.54$ & $17.62^{* * *}$ & $23.78 \pm 4.19$ & $130.93^{* * *}$ \\
\hline & Spouse & $25.51 \pm 3.55$ & & $25.86 \pm 3.45$ & & $25.27 \pm 3.59$ & \\
\hline & Non-spouse & $22.55 \pm 4.98$ & & $23.74 \pm 5.00$ & & $22.44 \pm 4.97$ & \\
\hline \multirow[t]{2}{*}{ ES } & National Health Insurance & $24.25 \pm 4.27$ & $38.17^{* * *}$ & $25.64 \pm 3.58$ & $12.98^{* * *}$ & $23.85 \pm 4.37$ & $33.23^{* * *}$ \\
\hline & Medical Aid & $22.58 \pm 5.60$ & & $24.09 \pm 5.02$ & & $21.96 \pm 5.72$ & \\
\hline \multirow[t]{2}{*}{ Hypertension } & No & $24.33 \pm 4.38$ & $5.07^{*}$ & $25.46 \pm 3.69$ & 0.43 & $23.88 \pm 4.54$ & 1.79 \\
\hline & Yes & $24.04 \pm 4.36$ & & $25.61 \pm 3.76$ & & $23.68 \pm 4.14$ & \\
\hline \multirow[t]{2}{*}{ DM } & No & $24.17 \pm 4.38$ & 0.12 & $25.50 \pm 3.79$ & 0.33 & $23.78 \pm 4.47$ & 0.29 \\
\hline & Yes & $24.13 \pm 4.34$ & & $25.65 \pm 3.51$ & & $23.69 \pm 4.46$ & \\
\hline \multirow[t]{2}{*}{ Hyperlipidemia } & No & $23.98 \pm 4.45$ & $41.59 * * *$ & $25.48 \pm 3.72$ & 2.33 & $23.49 \pm 4.56$ & $58.98^{* * *}$ \\
\hline & Yes & $25.07 \pm 3.83$ & & $26.07 \pm 3.73$ & & $24.93 \pm 3.83$ & \\
\hline \multirow[t]{2}{*}{ Stroke } & No & $24.18 \pm 4.36$ & 1.46 & $25.59 \pm 3.70$ & $5.23^{*}$ & $23.76 \pm 4.45$ & 0.16 \\
\hline & Yes & $23.83 \pm 4.63$ & & $24.45 \pm 3.97$ & & $23.63 \pm 4.82$ & \\
\hline \multirow[t]{3}{*}{ No. of comorbid diseases } & 0 & $24.37 \pm 4.45$ & 0.81 & $25.25 \pm 3.73$ & $12.97^{* * *}$ & $23.98 \pm 4.68$ & $33.23^{* * *}$ \\
\hline & $1-2$ & $24.11 \pm 4.36$ & & $26.70 \pm 3.61$ & & $23.62 \pm 4.45$ & \\
\hline & $\geq 3$ & $24.18 \pm 4.33$ & & $25.09 \pm 4.35$ & & $24.00 \pm 4.30$ & \\
\hline \multirow[t]{3}{*}{ Alcohol drinking } & Never & $23.84 \pm 4.47$ & $45.13^{* * *}$ & $25.15 \pm 4.23$ & $6.84^{* *}$ & $23.75 \pm 4.47$ & $10.85^{* * *}$ \\
\hline & Ceased drinking & $24.53 \pm 4.36$ & & $26.03 \pm 3.30$ & & $24.55 \pm 3.72$ & \\
\hline & Currently drinking & $25.49 \pm 3.53$ & & $25.22 \pm 3.79$ & & $22.33 \pm 5.25$ & \\
\hline \multirow[t]{3}{*}{ Smoking } & Never & $23.90 \pm 4.41$ & $40.43^{* * *}$ & $25.37 \pm 3.75$ & 0.43 & $23.77 \pm 4.44$ & 0.66 \\
\hline & Ceased smoking & $25.42 \pm 3.92$ & & $25.61 \pm 3.72$ & & $23.56 \pm 5.24$ & \\
\hline & Currently smoking & $25.02 \pm 4.21$ & & $25.57 \pm 3.68$ & & $22.97 \pm 5.35$ & \\
\hline \multirow[t]{2}{*}{ Exercise } & No & $23.29 \pm 4.78$ & $172.78^{* * *}$ & $24.68 \pm 4.41$ & $36.94 * * *$ & $22.98 \pm 4.80$ & $113.62^{* * *}$ \\
\hline & Yes & $24.91 \pm 3.83$ & & $26.05 \pm 3.13$ & & $24.51 \pm 3.98$ & \\
\hline
\end{tabular}

ES, economic status by health insurance type; DM, diabetes mellitus.

${ }^{+} p<0.1,{ }^{*} p<0.05,{ }^{* *} p<0.01,{ }^{* * *} p<0.001$. 
Table 3. Gender interaction in the effect of variables on cognitive function

\begin{tabular}{|c|c|c|c|c|}
\hline Variables & Source of variation & $\begin{array}{c}\text { Gender*Variable } \\
\text { F }\end{array}$ & $\begin{array}{c}\text { CV: Age } \\
\text { Gender*Variable } \\
\text { F }\end{array}$ & $\begin{array}{c}\text { CV: Education } \\
\text { Gender*Variable } \\
\text { F }\end{array}$ \\
\hline \multirow[t]{5}{*}{ Age } & (CV) Age & - & $687.45^{* * *}$ & - \\
\hline & (CV) Education & - & - & $683.20^{* * *}$ \\
\hline & Age & $10.14^{* * *}$ & - & $6.31^{* * *}$ \\
\hline & Gender & $44.68^{* * *}$ & $130.18^{* * *}$ & 2.56 \\
\hline & Gender*Age) & $1.86^{* *}$ & - & $1.11^{+}$ \\
\hline \multirow[t]{5}{*}{ Education period } & (CV) Age & - & $270.07^{* * *}$ & - \\
\hline & (CV) Education & - & - & $1,099.20^{* * *}$ \\
\hline & Education & $153.55^{* * *}$ & $104.97^{* * *}$ & - \\
\hline & Gender & 403.00 & $2.98^{+}$ & 0.09 \\
\hline & Gender*Education & $3.56^{*}$ & 1.82 & - \\
\hline \multirow[t]{5}{*}{ Depression } & (CV) Age & - & $743.92 * * *$ & - \\
\hline & (CV) Education & - & - & $1,038.37^{* * *}$ \\
\hline & Gender & $60.68^{* * *}$ & $53.46^{* * *}$ & $3.28^{+}$ \\
\hline & Depression & $10.93^{* * *}$ & $14.30^{* * *}$ & $8.03^{* * *}$ \\
\hline & Gender*Depression & 1.03 & 0.91 & 1.17 \\
\hline \multirow[t]{5}{*}{ Past occupation } & (CV) Age & - & $599.32^{* * *}$ & - \\
\hline & (CV) Education & - & - & $1,017.58^{* * *}$ \\
\hline & Gender & $32.58^{* * *}$ & $41.15^{* * *}$ & $5.81^{*}$ \\
\hline & Past occupation & $47.94^{* * *}$ & $17.35^{* * *}$ & $21.06^{* * *}$ \\
\hline & Gender*Past occupation & 0.39 & 1.16 & 0.17 \\
\hline \multirow[t]{5}{*}{ No. of social activities } & (CV) Age & - & $774.23^{* * *}$ & - \\
\hline & (CV) Education & - & - & $1,097.86^{* * *}$ \\
\hline & Gender & 2.96 & $6.08^{*}$ & 1.23 \\
\hline & No. of social activities & $2.99^{* * *}$ & $8.00^{* * *}$ & $4.82^{* * *}$ \\
\hline & Gender* No. of social activities & $1.94^{* *}$ & 1.17 & 1.38 \\
\hline \multirow[t]{5}{*}{ Religion } & (CV) Age & - & $700.72^{* * *}$ & - \\
\hline & (CV) Education & - & & $1,063.98^{* * *}$ \\
\hline & Gender & $40.60 * * *$ & $40.90^{* * *}$ & 0.15 \\
\hline & Religion & $7.86^{* * *}$ & $9.89^{* * *}$ & $5.84^{* * *}$ \\
\hline & Gender*Religion & 2.11 & 1.64 & $2.12^{+}$ \\
\hline \multirow[t]{5}{*}{ Cohabitant } & (CV) Age & - & $453.65^{* * *}$ & - \\
\hline & (CV) Education & - & - & $896.60^{* * *}$ \\
\hline & Gender & $40.85^{* * *}$ & $44.52^{* * *}$ & $3.69^{* * *}$ \\
\hline & Cohabitant & $66.10^{* * *}$ & $23.90^{* * *}$ & $32.10^{* * *}$ \\
\hline & Gender*Cohabitant & $4.15^{*}$ & 0.04 & 0.86 \\
\hline \multirow[t]{5}{*}{ ES } & (CV) Age & - & $677.74^{* * *}$ & \\
\hline & (CV) Education & - & & $1,108.41^{* * *}$ \\
\hline & Gender & $43.74 * * *$ & $26.52^{* * *}$ & 0.30 \\
\hline & ES & $33.86^{* * *}$ & $34.19^{* * *}$ & $39.40^{* * *}$ \\
\hline & Gender*ES & 3.18 & 0.52 & 0.45 \\
\hline \multirow[t]{5}{*}{ Hypertension } & (CV) Age & - & $697.34^{* * *}$ & \\
\hline & (CV) Education & - & & $1,095.40^{* * *}$ \\
\hline & Gender & $140.78^{* * *}$ & $137.48^{* * *}$ & 0.05 \\
\hline & Hypertension & 0.04 & $5.33^{*}$ & 0.51 \\
\hline & Gender*Hypertension & 1.40 & 0.89 & 0.01 \\
\hline
\end{tabular}


Table 3. Continued

\begin{tabular}{|c|c|c|c|c|}
\hline Variables & Source of variation & $\begin{array}{c}\text { Gender*Variable } \\
\text { F }\end{array}$ & $\begin{array}{c}\text { CV: Age } \\
\text { Gender*Variable } \\
\text { F }\end{array}$ & $\begin{array}{c}\text { CV: Education } \\
\text { Gender*Variable } \\
\text { F }\end{array}$ \\
\hline \multirow[t]{5}{*}{ Hyperlipidemia } & (CV) Age & - & $657.99 * * *$ & - \\
\hline & (CV) Education & - & - & $1,079.49 * * *$ \\
\hline & Gender & $42.48^{* * *}$ & $32.99 * * *$ & 1.03 \\
\hline & Hyperlipidemia & $17.75^{* * *}$ & $6.90^{* *}$ & $12.20^{* * *}$ \\
\hline & Gender*Hyperlipidemia & $3.08^{+}$ & $4.28^{*}$ & $3.06^{+}$ \\
\hline \multirow[t]{5}{*}{ Stroke } & (CV) Age & - & $689.25^{* * *}$ & - \\
\hline & (CV) Education & - & - & $1,098.46^{* * *}$ \\
\hline & Gender & $15.76^{* * *}$ & $16.28^{* * *}$ & 0.90 \\
\hline & Stroke & $3.69^{+}$ & $5.87^{*}$ & $3.81^{+}$ \\
\hline & Gender*Stroke & 2.27 & 1.26 & 0.84 \\
\hline \multirow[t]{5}{*}{ No. of comorbid diseases } & (CV) Age & - & $696.34^{* * *}$ & - \\
\hline & (CV) Education & - & - & $1,097.85^{* * *}$ \\
\hline & Gender & $105.45^{* * *}$ & $93.99 * * *$ & 0.78 \\
\hline & No. of comorbid diseases & 0.17 & 2.07 & 0.56 \\
\hline & Gender*No. of comorbid diseases & $2.74^{*}$ & $3.57^{*}$ & $2.12^{+}$ \\
\hline \multirow[t]{5}{*}{ Alcohol drinking } & (CV) Age & - & $667.61^{* * *}$ & - \\
\hline & (CV) Education & - & - & $1,093.93^{* * *}$ \\
\hline & Gender & $87.52^{* * *}$ & $95.52^{* * *}$ & 1.01 \\
\hline & Alcohol drinking & $16.39^{* * *}$ & $6.52^{* * *}$ & $14.83^{* * *}$ \\
\hline & Gender*Alcohol drinking & $4.62^{* *}$ & $4.08^{*}$ & 3.01 \\
\hline \multirow[t]{5}{*}{ Smoking } & (CV) Age & - & $689.75^{* * *}$ & - \\
\hline & (CV) Education & - & - & $1,100.74^{* * *}$ \\
\hline & Gender & $38.97^{* * *}$ & $29.03^{* * *}$ & 1.04 \\
\hline & Smoking & 0.27 & 1.60 & 0.67 \\
\hline & Gender*Smoking & 0.89 & 0.01 & 1.52 \\
\hline \multirow[t]{5}{*}{ Exercise } & (CV) Age & - & $616.97^{* * *}$ & - \\
\hline & (CV) Education & - & - & $1,003.71^{* * *}$ \\
\hline & Gender & $119.45^{* * *}$ & $107.50 * * *$ & 0.27 \\
\hline & Exercise & $94.69^{* * *}$ & $61.56^{* * *}$ & $46.25^{* * *}$ \\
\hline & Gender*Exercise & 0.31 & 0.11 & 0.01 \\
\hline
\end{tabular}

$\mathrm{CV}$, co-variable; $\mathrm{ES}$, economic status by health insurance type.

${ }^{+} p<0.1,{ }^{*} p<0.05,{ }^{* *} p<0.01,{ }^{* * *} p<0.001$.

factors affecting cognitive impairment were analyzed using hierarchical regression. The significance level for the statistical test was set to 0.05 .

\section{RESULTS}

\section{Distribution of cognitive function-related variables according to gender}

We identified differences in the distribution of age, depression, education period, economic status, cohabitant, social life, history of disease, and lifestyle habits among the participants according to gender (Table 1).

\section{Gender comparison of cognitive function score according to each variable}

The cognitive function scores by gender were $25.53 \pm 3.72$ points in men and $23.76 \pm 4.47$ in the women, showing a significant difference $(\mathrm{F}=54.31 ; \mathrm{p}<0.001)$.

There were significant differences in cognitive function scores in the whole group, men participants and women participants according to age, education, depression, past occupation, economic status, cohabitant, drinking and exercise. There was no significant difference in cognitive function score according to the presence or absence of diabetes in the whole group, men and women participants (Table 2).

However, the cognitive function scores differed between the whole group, men and women participants according to number 
of social activities, religion, hypertension, hyperlipidemia, stroke, number of comorbid diseases and smoking (Table 2).

\section{Gender interaction with cognitive function-related factors}

Two-way ANOVA was performed to determine the interaction of each variable affecting cognitive function with gender using gender and each variable as independent variables. As a result, the variables that interacted with gender in affecting cognitive function scores included age $(\mathrm{F}=1.86 ; \mathrm{P}<0.01)$, education period $(\mathrm{F}=3.56 ; \mathrm{p}<0.05)$, social activities $(\mathrm{F}=1.94 ; \mathrm{p}<0.05)$, cohabitant $(\mathrm{F}=4.15 .15 ; \mathrm{p}<0.05)$, number of comorbid diseases $(\mathrm{F}=2.74$; $\mathrm{p}<0.05)$ and drinking $(\mathrm{F}=4.62, \mathrm{p}<0.01)$. Hypertension, stroke and smoking did not show gender interactions and the main effects were not significant, showing that hypertension, stroke and smoking did not affect cognitive function. Depression, past occupation, religion, economic status, hyperlipidemia and exercise showed significant main effects without interactions with gender (Table 3).

In assess for possible interactive variables after controlling for gender and other variables, two-way ANOVA between gender and each variable was performed by using age and education period as co-variates. When gender interactions with each variable and main effects when only age was controlled were identified, the results showed that hyperlipidemia, the number of comorbid diseases and drinking had significant interactions with gender $(\mathrm{F}=4.28, \mathrm{p}<0.05 ; \mathrm{F}=3.57 ; \mathrm{p}<0.05, \mathrm{~F}=4.08 ; \mathrm{p}<0.05)$, and all interactions with gender disappeared when only education was controlled (Table3).

\section{Factors affecting cognitive function according to gender}

The aptness of the regression equation was found to be acceptable for men with a tolerance of 0.730-0.970, variance inflation factor (VIF) of 1.031-1.369 and Durbin-Watson statistic of 1.898 and for women with a tolerance of $0.732-0.973$, VIF of 1.028-1.366 and Durbin-Watson statistic of 1.835 .

To identify factors affecting cognitive function when controlling for education and age, education and age were first input and then the remaining variables were input to perform hierarchical regression analysis by gender (Table 4 ).

For the male participants, the effect of education $(\beta=0.30)$ and

Table 4. Factors influencing cognitive function according to gender

\begin{tabular}{|c|c|c|c|c|c|c|c|c|c|c|c|}
\hline \multirow{2}{*}{ Gender } & \multirow{2}{*}{ Variables } & \multicolumn{5}{|c|}{ Model 1} & \multicolumn{5}{|c|}{ Model 2} \\
\hline & & B & SE & $\beta$ & $\mathrm{t}$ & p-value & B & SE & $\beta$ & $\mathrm{t}$ & p-vlaue \\
\hline \multirow[t]{11}{*}{ Men } & (Constant) & 28.93 & 1.26 & & 23.04 & $<0.001$ & 28.39 & 1.40 & & 20.28 & $<0.001$ \\
\hline & Education period & 1.38 & 0.13 & 0.30 & 10.65 & $<0.001$ & 1.20 & 0.13 & 0.26 & 9.44 & $<0.001$ \\
\hline & Age & 0.09 & 0.02 & -0.17 & -6.10 & $<0.001$ & -0.10 & 0.02 & -0.19 & -6.51 & $<0.001$ \\
\hline & Depression & & & & & & -0.17 & 0.03 & -0.16 & -5.43 & $<0.001$ \\
\hline & Cohabitant: non- spouse & & & & & & -1.32 & 0.33 & -0.11 & -3.99 & $<0.001$ \\
\hline & No. of social activities & & & & & & 0.56 & 0.16 & 0.10 & 3.51 & $<0.001$ \\
\hline & ES & & & & & & -1.23 & 0.41 & -0.09 & -3.03 & 0.002 \\
\hline & Exercise & & & & & & 0.60 & 0.21 & 0.08 & 2.80 & 0.005 \\
\hline & Stroke & & & & & & -1.05 & 0.48 & -0.06 & -2.22 & 0.03 \\
\hline & Religion & & & & & & 0.42 & 0.21 & 0.06 & 2.03 & 0.04 \\
\hline & & \multicolumn{5}{|c|}{$\begin{array}{l}\quad F(p)=89.35^{* * *} \\
\text { adjusted } R^{2}=0.14, \Delta R^{2}=0.14\end{array}$} & \multicolumn{5}{|c|}{$\begin{array}{c}\mathrm{F}=18.83^{* * *} \\
\mathrm{R}^{2}=0.23, \text { adjusted } \mathrm{R}^{2}=0.21, \Delta \mathrm{R}^{2}=0.09\end{array}$} \\
\hline \multirow[t]{12}{*}{ Women } & (Constant) & 31.39 & 0.79 & & 39.58 & $<0.001$ & 31.12 & 0.90 & & 34.72 & $<0.001$ \\
\hline & Education period & 1.92 & 0.08 & 0.36 & 23.28 & $<0.001$ & 1.72 & 0.08 & 0.32 & 20.96 & $<0.001$ \\
\hline & Age & -0.14 & 0.01 & -0.24 & -15.43 & $<0.001$ & -0.14 & 0.01 & -0.23 & -13.38 & $<0.001$ \\
\hline & Depression & & & & & & -0.21 & 0.02 & -0.16 & -10.93 & $<0.001$ \\
\hline & Cohabitant: non- spouse & & & & & & -0.74 & 0.17 & -0.08 & -4.39 & $<0.001$ \\
\hline & ES & & & & & & -1.30 & 0.28 & -0.06 & -4.65 & $<0.001$ \\
\hline & Hyperlipidemia & & & & & & 0.63 & 0.19 & 0.05 & 3.29 & 0.001 \\
\hline & No. of social activities & & & & & & 0.31 & 0.10 & 0.04 & 3.08 & 0.002 \\
\hline & Exercise & & & & & & 0.33 & 0.13 & 0.04 & 2.55 & 0.01 \\
\hline & Religion & & & & & & -0.33 & 0.14 & -0.03 & -2.37 & 0.02 \\
\hline & Stroke & & & & & & -0.63 & 0.30 & -0.03 & -2.09 & 0.04 \\
\hline & & \multicolumn{5}{|c|}{$\begin{array}{l}\quad F(p)=635.99^{* * *} \\
\text { adjusted } R^{2}=0.25, \Delta R^{2}=0.25\end{array}$} & \multicolumn{5}{|c|}{$\begin{array}{l}\mathrm{F}=99.00^{* * *} \\
\text { djusted } \mathrm{R}^{2}=0.31, \Delta \mathrm{R}^{2}=0.06\end{array}$} \\
\hline
\end{tabular}

$\mathrm{SE}$, standard error; ES, economic status by health insurance type.

*** $p<0.001$. 
age $(\beta=-0.17)$ on cognitive function scores was $R^{2}=0.14$. The effects of depression $(\beta=-0.16)$, living with non-spouse $(\beta=-0.11)$, social activities $(\beta=0.10)$, economic status $(\beta=-0.09)$, exercise $(\beta=0.08)$, presence or absence of stroke $(\beta=0.06)$ and presence or absence of religion $(\beta=0.06)$ on cognitive function score were low but significant.

For the female participants, the effect of education period $(\beta=0.36)$ and age $(\beta=-0.24)$ on cognitive function score was $R^{2}=0.25$. Depression $(\beta=-0.16)$, living with non-spouse $(\beta=-0.08)$, hyperlipidemia $(\beta=0.05)$, economic status $(\beta=-0.06)$, social activities $(\beta=$ $0.04)$, exercise $(\beta=0.04)$, the presence or absence of religion $(\beta=$ $-0.03)$ and presence or absence of stroke $(\beta=-0.03)$ had significant effects on cognitive function score.

\section{DISCUSSION}

The proportion of elderly participants aged 85 years or older was higher among women, and the proportion of those with education period of less than 3 years was higher among women, which were similar to the distribution of age and education period in the studies by Park et al. [10], Kim et al. [14], and Park et al. [17] of community-dwelling elderly people. Furthermore, the results of the present study found that the proportion of those who engaged in regular exercise was higher among men, which has been reported as a cognitive function protective factor in previous studies $[18,19]$, and smoking and drinking, which have been reported as cognitive function risk factors were more frequent in the men. From these results, it can be predicted that the difference in gender distribution of cognitive function protective factors or risk factors, or the relationship between those factors and gender might affect the gender differences in cognitive function.

Looking at cognitive function scores according to cognitive variables, possible risk factors for both genders included short education period, high depression, 'no past occupation' 'living with nonspouse family members', 'economic status' and 'no exercise. Depression has been considered as a predictor of cognitive function in many previous studies [18,20,21]; it is therefore an important factor for maintaining cognitive function, and it is thought that continuous attention should be paid to depression to prevent decline in cognitive function. Living with family in terms of cohabitation type is accepted as a cognitive protective factor. Fratiglioni et al. [22] and Park et al. [17] reported that elderly individuals living alone had a relatively low cognitive function compared to those living with family, and explained that such results were due to the fact that elderly people living alone had a lack of emotional and cognitive stimulation and sense due to isolated life with little family and social ties. However, the present study found that elderly individuals living with their spouse had the highest cognitive function scores, followed by elderly people living alone and elderly people living with non-spouse family. This suggests that the cohabitation type of Korean elderly people is also changing to be couple-oriented, and living with children other than spouse is considered not to be protective of cognitive function compared to living alone. Continuous observational studies regarding cohabitation-related factors in Korean elderly people are needed.

Regarding drinking among lifestyle factors, 'never-drinkers' and 'those who stopped drinking' among the man participants had a lower cognitive function than the current drinkers, whereas the 'current drinkers' in the women group were found to have the lowest cognitive function score ( $22.33 \pm 5.25$ points), suggesting that the effects of drinking on cognitive function may differ according to gender. The studies by Park \& Song [8], Shin et al. [23], and Kim \& Shim [24] have reported that drinking was associated with cognitive function; however, Topiwala et al. [25] reported through a systematic literature review that drinking was not associated with dementia. The results of the present study also showed that the effects of drinking on cognitive function were not clear. In this regard, it is necessary to investigate the degree and duration of drinking and to examine their relationship with other confounding variables. Men and women participants who exercised were found to have high cognitive function in the present study, which was consistent with the results of previous studies investigating the effects of physical activity on cognitive impairment [17,21]. Therefore, the present study confirmed that exercise was protective of cognitive function.

Vascular risk factors such as hypertension and diabetes are estimated to be risk factors for the progression of cognitive decline and transition from mild cognitive impairment to dementia $[6,21$, 26]. But the results of the present study showed that there was no difference in cognitive function according to diabetes and stroke, which were inconsistent with the results of previous studies $[8,17$, $21]$. Further studies are needed to investigate the degree of hypertension and duration of disease.

The present study showed that only among woman participants, there were differences in cognitive function according to hyperlipidemia and there were a higher cognitive function in the woman participants with hyperlipidemia than those without hyperlipidemia, suggesting that hyperlipidemia may have a positive effect on cognitive function in women. In a study by Park et al. [17], hyperlipidemia was reported as a protective factor for mild cognitive impairment because it showed a negative relative risk and odds ratio, which were similar to the results of a study by Vidoni et al. [27] reporting that a low cholesterol or a low BMI acts as a risk factor for cognitive impairment.

There was a significant difference in cognitive function according to stroke only in man participants $(\mathrm{F}=5.23 ; \mathrm{p}<0.05)$, showing that stroke was a risk factor for cognitive function among man participants, and this finding was similar to the results of a study by Park et al. [17] revealing that stroke had a greater relative risk in men than women.

The cognitive function according to smoking status was not different and the lowest cognitive function score was observed in never-smokers. These findings are similar to the results of studies by Shin et al.[23], and other researchers [10,23]. However, Kim [7] and Rakesh et al. [21] have reported that smoking was a risk factor for cognitive function. More detailed studies on the effects of 
smoking on cognitive function are thus needed.

Whether the gender difference in cognitive function as found in the present study was simply due to gender difference or gender difference in cognitive function-related factors or due to the results of interaction between those factors and gender is discussed as follows. Cognitive function scores did not differ according to gender when the education period was the same, and differences in cognitive function were found to be due to education period rather than age in the present study. This finding was consistent with the results of a study by Kim [7] and Park et al. [10] indicating that education was the most influential variable on cognitive function. Lin et al. [9] described that cognitive impairment and dementia were more likely to affect women than men, but this could be seen as a difference in age distribution. However, the results of the present study can be interpreted based on the explanation that cognitive impairment might progress faster as education period is shorter and the resulting cognitive simulation is lacking [28]. This is in line with the results of a study by Petersen et al. [29] stating that men might be relatively slower to progress from mild cognitive impairment to dementia because they have a relatively longer education period than women.

In addition, there were gender interactions with age, education period, social activities, cohabitant, drinking and the number of comorbid diseases among cognitive function-related variables in affecting cognitive function. However, when age was controlled, social activities and cohabitant showed no gender interaction, and only significant main effects were observed, suggesting that the difference in gender and age might affect cognitive function in relation to social activities and cohabitant. Meanwhile, drinking and the number of comorbid diseases showed significant interactions with gender even when age was controlled. However, when education was controlled, these variables did not show interactions with gender and the main effects of each variable were significant. The significant interactions of these variables with gender are interpreted to be due to gender differences in education period.

Finally, as a result of regression analysis it was found that depression in addition to education and age had a great effect on cognitive function in both gender participants (Table 4). The variable that differed between man and woman participants was found to be hyperlipidemia and was found to be a significant variable only in the woman participants. Therefore, further studies are needed to determine whether hyperlipidemia itself has a protective function for cognitive function, or whether it is related to education period and economic status in addition to age.

In conclusion, the results of the present study showed that fender differences in cognitive function was due to differences in education period. And factors affecting cognitive function were same for men and women, but, hyperlipidemia was added as a factor affecting cognitive function in the female participants.

Further studies are needed to investigate factors affecting cognitive function, the interactions with education and the combined effects. It is thought that it is effective to provide elderly individuals with interventional programs for improving cognitive function by segmenting them by education period and age.

\section{CONFLICT OF INTEREST}

The authors have no conflicts of interest to declare for this study.

\section{SUPPLEMENTARY MATERIAL}

Supplementary material (Korean version) is available at http:// www.e-epih.org/.

\section{ORCID}

Miwon Kim: http://orcid.org/0000-0002-4142-1202; Jeongn-Mo Park: http://orcid.org/0000-0002-5354-2335

\section{REFERENCES}

1. Korean Statistics Information Service. Estimated future population; 2017 [cited 2017 Oct 30]. Available from: http://kosis.kr/ statHtml/statHtml.do?orgId = 101\&tblid = DT_1BPA002\&conn_ path $=$ I2 (Korean).

2. Han J, Kang H. The relationship between functional physical fitness and mild cognitive impairment in older adults. Korean J Sports Med 2015;33:102-109 (Korean).

3. Kim KW, Kim YJ, Kim, BN, Kim HN, Choi HJ, You JY, et al. The 3rd dementia management integration plant. Seongnam: Seoul National University Bundang Hospital; 2015, p. 25-26 (Korean).

4. Prince M, Bryce R, Albanese E, Wimo A, Ribeiro W, Ferri CP. The global prevalence of dementia: a systematic review and metaanalysis. Alzheimers Dement 2013;9:63-75.

5. Ministry of Health and Welfare. 3rd comprehensive plan for dementia management (16'-20'); 2015 [cited 2017 Jul 15]. Available from http://www.mohw.go.kr/react/jb/sjb030301vw.jsp?PAR_MENU_ ID $=03 \&$ MENU_ID $=0319 \&$ CONT_SEQ $=330876 \&$ page $=1$ (Korean).

6. Li W, Wang T. Xiao S. Type 2 diabetes mellitus might be a risk factor for mild cognitive impairment progressing to Alzheimer's disease. Neuropsychiatr Dis Treat 2016;12:2489-2495.

7. Kim EJ. Factors influencing cognitive impairment of the elderly residents. J East West Nurs Res 2010;16:122-130 (Korean).

8. Park HK, Song HJ. Predictors of cognitive function decline of elderly: using living conditions and welfare needs of older Korean persons panel data. Korean J Health Serv Manag 2016;10:147-158 (Korean).

9. Lin JS, O'Connor E, Rossom RC, Perdue LA, Burda BU, Thompson $\mathrm{M}$, et al. Screening for cognitive impairment in older adults: an evidence update for the U.S. Preventive Services Task Force [cited 2017 May 2]. Available from: https://www.ncbi.nlm.nih. gov/pubmedhealth/PMH0063382/.

10. Park JS, Lee YW, Kim HS. Cognitive impairment risk factors in elders. J Korean Gerontol Nurs 2015;17:121-130 (Korean).

11. Barnes DE, Yaffe K. The projected effect of risk factor reduction 
on Alzheimer's disease prevalence. Lancet Neurol 2011;10:819828.

12. Oh E, Lee AY. Mild cognitive impairment. J Korean Neurol Assoc 2016;34:167-175 (Korean).

13. Koran ME, Wagener M, Hohman TJ; Alzheimer's Neuroimaging Initiative. Sex differences in the association between $\mathrm{AD}$ biomarkers and cognitive decline. Brain Imaging Behav 2017;11:205-213.

14. Kim KW, Kim MH, Kim BJ, Kim JR, Kim TH, Moon SW, et al. Standardization of dementia diagnosis tool. Seongnam: Seoul National University Bundang Hospital; 2009, p. 29-53 (Korean).

15. Yesavage JA, Sheikh JI. Geriatric depression scale (GDS): recent evidence and development of a shorter version. Clin Gerontol 1986;5:165-173.

16. Ki BS. A preliminary study for the standardization of geriatric depression scale short form-Korea version. J Korean Neuropsychiatr Assoc 1996;35:298-307 (Korean).

17. Park M, Sung MR, Kim SK, Lee DY. Comparison of demographic characteristics, comorbidity, and health habits of older adults with mild cognitive impairment and older adults with normal cognitive function. J Korean Acad Nurs 2014;44:351-360 (Korean).

18. Park HJ. Factors analysis and prediction models of MCI using the KLoSA [dissertation]. Busan: Pusan National University; 2016 (Korean).

19. Gomez-Pinilla F, Hillman C. The influence of exercise on cognitive abilities. Compr Physiol 2013;3:403-428.

20. Boyle PA, Willson RS. Risk factors for Alzheimer's disease. In: Ames D, O'Brien JT, Burns A, editors. Dementia, fifth edition. New York: CRC Press; 2017, p. 447-452.

21. Rakesh G, Szabo ST, Alexopoulos GS, Zannas AS. Strategies for dementia prevention: latest evidence and implications. Ther Adv Chronic Dis 2017;8:121-136.
22. Fratiglioni L, Paillard-Borg S, Winblad B. An active and socially integrated lifestyle in late life might protect against dementia. Lancet Neurol 2004;3:343-353.

23. Shin KR, Kang Y, Jung D, Kim M, Kim J, Kim M, et al. Prevalence and characteristics of mild cognitive impairment in the community-dwelling elderly compared to elderly with normal cognitive function. Korean J Adult Nurs 2011;23:40-49 (Korean).

24. Kim YO, Shim MS. Cognitive functions, instrumental activities of daily living, depression and quality of life in the elderly with mild cognitive impairment. J Korean Public Health Nurs 2015; 29:219-230 (Korean).

25. Topiwala A, Allan CL, Valkanova V, Zsoldos E, Filippini N, Sexton $\mathrm{C}$, et al. Moderate alcohol consumption as risk factor for adverse brain outcomes and cognitive decline: longitudinal cohort study. BMJ 2017;357:j2353.

26. Schneider BC, Gross AL, Bangen KJ, Skinner JC, Benitez A, Glymour MM, et al. Association of vascular risk factors with cognition in a multiethnic sample. J Gerontol B Psychol Sci Soc Sci 2015; 70:532-544.

27. Vidoni ED, Townley RA, Honea RA, Burns JM; Alzheimer's Disease Neuroimaging Initiative. Alzheimer disease biomarkers are associated with body mass index. Neurology 2011;77:1913-1920.

28. Ganguli M, Snitz BE, Lee CW, Vanderbilt J, Saxton JA, Chang CC. Age and education effects and norms on a cognitive test battery from a population-based cohort: the Monongahela-Youghiogheny Healthy Aging Team. Aging Ment Health 2010;14:100107.

29. Petersen RC, Roberts RO, Knopman DS, Geda YE, Cha RH, Pankratz VS, et al. Prevalence of mild cognitive impairment is higher in men. The Mayo Clinic Study of Aging. Neurology 2010;75:889897. 